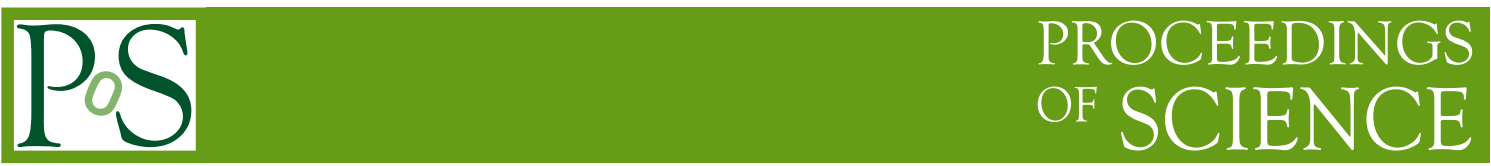

\title{
Utilising $B \rightarrow \pi K$ Decays at the High-Precision Frontier
}

\section{R. Jaarsma*}

Nikhef, Science Park 105, 1098 XG Amsterdam, Netherlands

E-mail: rjaarsma@nikhef.nI

$B \rightarrow \pi K$ decays have received a lot of attention over the last two decades, with puzzling patterns in the previous data. They form a particularly interesting set of decays as they are dominated by QCD penguin topologies. Electroweak penguin amplitudes also play a significant role, giving a contribution at the level of the tree topologies. We find a discrepancy in the correlation between the CP asymmetries of $B_{d}^{0} \rightarrow \pi^{0} K_{\mathrm{S}}$. A modified electroweak penguin sector offers an attractive avenue for new particles to enter. We provide a new method to determine the electroweak penguin parameters, and apply it to current data for charged $B \rightarrow \pi K$ decays. It uses an isospin relation and requires only $S U(3)$ input to fix a normalization. A central role is played by the mixing-induced $\mathrm{CP}$ asymmetry of $B_{d}^{0} \rightarrow \pi^{0} K_{\mathrm{S}}$. The implementation of our strategy in the high-precision era of $B$-physics has the exciting potential to establish New Physics in the electroweak penguin sector.

The International Conference on B-Physics at Frontier Machines - BEAUTY2018

6-11 May, 2018

La Biodola, Elba Island, Italy

* Speaker. 


\section{Introduction}

Decays of the type $B \rightarrow \pi K$ have been in the spotlight for over two decades ([1,2] and references therein). This is a particularly interesting class of decays because the leading contributions come from QCD penguin topologies; the tree topologies are suppressed by the CKM matrix element $V_{u b}$. Moreover, electroweak (EW) penguin amplitudes give contributions at the same level as the tree topologies.

The decay $B_{d}^{0} \rightarrow \pi^{0} K_{\mathrm{S}}$ is the only $B \rightarrow \pi K$ channel with a mixing-induced CP asymmetry. Moreover, all $B \rightarrow \pi K$ decays may have direct $\mathrm{CP}$ violation. The correlation between the $\mathrm{CP}$ asymmetries of the $B_{d}^{0} \rightarrow \pi^{0} K_{\mathrm{S}}$ mode has revealed a discrepancy in the past, which could be explained by a modified EW penguin sector [2]. We have a fresh look at this correlation, and present a new method to pin down the parameters governing the EW penguin contributions [3].

\section{The $B \rightarrow \pi K$ System}

The EW penguin topologies contributing to $B_{d}^{0} \rightarrow \pi^{-} K^{+}$and $B^{+} \rightarrow \pi^{+} K^{0}$ are colour-suppressed and play a minor role. On the other hand, the $B_{d}^{0} \rightarrow \pi^{0} K^{0}$ and $B^{+} \rightarrow \pi^{0} K^{+}$channels have also contributions from colour-allowed EW penguin toplogies. These effects are described by the following parameter, which can be calculated using the $S U(3)$ flavour symmetry $[4,1]$ :

$$
q e^{i \phi} e^{i \omega} \equiv-\left(\frac{\hat{P}_{E W}+\hat{P}_{E W}^{\mathrm{C}}}{\hat{T}+\hat{C}}\right) \stackrel{\mathrm{SM}}{=} \frac{-3}{2 \lambda^{2} R_{b}}\left(\frac{C_{9}+C_{10}}{C_{1}+C_{2}}\right) R_{q}=(0.68 \pm 0.05) R_{q} .
$$

Here $\phi(\omega)$ is a CP-violating (CP-conserving) phase, and $\hat{P}_{E W}(\hat{T})$ and $\hat{P}_{E W}^{\mathrm{C}}(\hat{C})$ are colour-allowed and colour-suppressed EW penguin (tree) amplitudes, respectively. Note that $\omega$ vanishes in the $S U$ (3) limit, and that its smallness is a model-independent result [5]. Furthermore, $\lambda \equiv\left|V_{u s}\right|=0.22$, $R_{b}$ is a side of the unitarity triangle (UT), and the $C_{i}$ are Wilson coefficients. The deviation of $R_{q}$ from 1 parametrizes $S U$ (3)-breaking corrections. We will use $R_{q}=1.0 \pm 0.3$, while progress in lattice QCD can bring the uncertainty down to \pm 0.05 in the future [2].

The hadronic parameters that describe the tree and QCD penguin topologies can be determined using $B \rightarrow \pi \pi$ data, where contributions from EW penguins are tiny, employing the $S U(3)$ flavour symmetry [1, 2]. Allowing for non-factorizable $S U$ (3)-breaking corrections of $20 \%$ we obtain [3]

$$
r_{\mathrm{c}} e^{i \delta_{\mathrm{c}}} \equiv \frac{\hat{T}+\hat{C}}{P^{\prime}}=(0.17 \pm 0.06) e^{i(1.9 \pm 23.9)^{\circ}}, \quad r e^{i \delta} \equiv \frac{\hat{T}-\hat{P}_{t u}}{P^{\prime}}=(0.09 \pm 0.03) e^{i(28.6 \pm 21.4)^{\circ}},
$$

where $\hat{P}_{t u}$ is the difference between QCD penguin amplitudes with $t$ and $u$ quarks, and $P^{\prime} \propto P_{t c}$. In an analysis of $B_{d, s} \rightarrow \pi \pi, K K, \pi K$ modes, no indications of anomalously large non-factorizable $S U$ (3)-breaking corrections were found [6].

The direct CP asymmetries $A_{\mathrm{CP}}^{f} \equiv\left(\left|\bar{A}_{f}\right|^{2}-\left|A_{f}\right|^{2}\right) /\left(\left|\bar{A}_{f}\right|^{2}+\left|A_{f}\right|^{2}\right)$, as well as the branching ratios, are ingredients of a sum rule [7], which vanishes in the SM up to corrections of $\mathscr{O}\left(r_{(\mathrm{c})}^{2}\right)$ [3]. The current experimental data [8] are in agreement with the SM pattern. Since the uncertainty of $A_{\mathrm{CP}}^{\pi^{0} K^{0}}$ is still large, we use the sum rule to predict $A_{\mathrm{CP}}^{\pi^{0} K^{0}}=-0.14 \pm 0.03$ [3].

The mixing-induced CP asymmetry $S_{\mathrm{CP}}^{\pi^{0} K \mathrm{~S}}$ enters the time-dependent rate asymmetry as

$$
\frac{\Gamma\left(\bar{B}_{d}^{0}(t) \rightarrow \pi^{0} K_{\mathrm{S}}\right)-\Gamma\left(B_{d}^{0}(t) \rightarrow \pi^{0} K_{\mathrm{S}}\right)}{\Gamma\left(\bar{B}_{d}^{0}(t) \rightarrow \pi^{0} K_{\mathrm{S}}\right)+\Gamma\left(B_{d}^{0}(t) \rightarrow \pi^{0} K_{\mathrm{S}}\right)}=A_{\mathrm{CP}}^{\pi^{0} K_{\mathrm{S}}} \cos \left(\Delta M_{d} t\right)+S_{\mathrm{CP}}^{\pi^{0} K_{\mathrm{S}}} \sin \left(\Delta M_{d} t\right),
$$



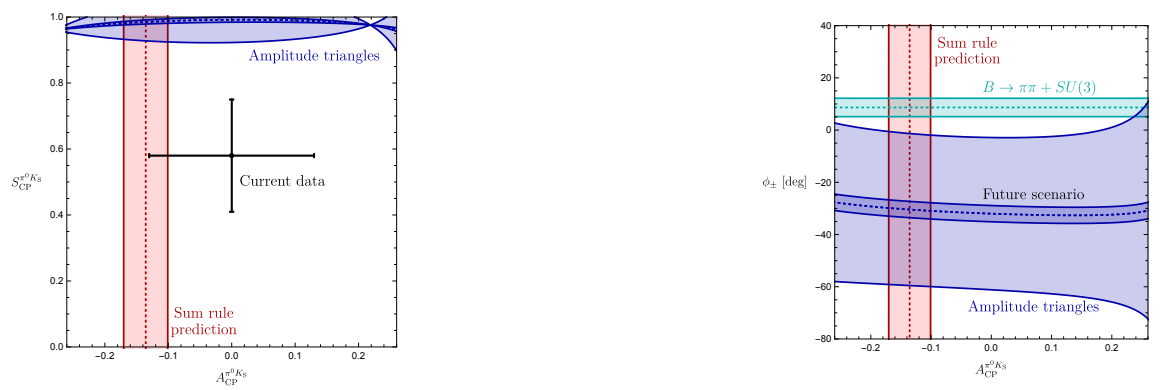

Figure 1: Left: Correlation between the CP asymmetries of $B_{d}^{0} \rightarrow \pi^{0} K_{\mathrm{S}}$. Right: $\phi_{ \pm}$as a function of $A_{\mathrm{CP}}^{\pi^{0} K_{\mathrm{S}}}$.

where $\Delta M_{d}$ is the mass difference between the $B_{d}$ mass eigenstates. We have

$$
S_{\mathrm{CP}}^{\pi^{0} K_{\mathrm{S}}}=\sin \left(\phi_{d}-\phi_{00}\right) \sqrt{1-\left(A_{\mathrm{CP}}^{\pi^{0} K_{\mathrm{S}}}\right)^{2}}
$$

where $\phi_{d}=(43.2 \pm 1.8)^{\circ}$ is the CP-violating $B_{d}^{0}-\bar{B}_{d}^{0}$ mixing phase [2]. The key quantity is the angle $\phi_{00} \equiv \arg \left(\overline{\mathrm{A}}_{00} \mathrm{~A}_{00}^{*}\right)$ between $A_{00} \equiv A\left(B_{d}^{0} \rightarrow \pi^{0} K^{0}\right)$ and its CP-conjugate $\bar{A}_{00}$, which can be expressed in terms of the hadronic parameters in Eq. (2.2) as follows [3]:

$$
\tan \phi_{00}=2\left(r \cos \delta-r_{\mathrm{c}} \cos \delta_{\mathrm{c}}\right) \sin \gamma+2 r_{\mathrm{c}}\left(\cos \delta_{\mathrm{c}}-2 \tilde{a}_{\mathrm{C}} / 3\right) q \sin \phi+\mathscr{O}\left(r_{(\mathrm{c})}^{2}\right) .
$$

Here $\tilde{a}_{\mathrm{C}} \equiv a_{\mathrm{C}} \cos \left(\Delta_{\mathrm{C}}+\delta_{\mathrm{c}}\right)$ parametrizes the small colour-suppressed EW penguin contributions.

\section{Correlations Between the CP Asymmetries of $B_{d}^{0} \rightarrow \pi^{0} K_{\mathrm{S}}$}

The amplitudes of the $B \rightarrow \pi K$ decays satisfy the following isospin relation [1,2]:

$$
\begin{aligned}
3 A_{3 / 2} & \equiv \sqrt{2} A\left(B_{d}^{0} \rightarrow \pi^{0} K^{0}\right)+A\left(B_{d}^{0} \rightarrow \pi^{-} K^{+}\right)=\sqrt{2} A\left(B^{+} \rightarrow \pi^{0} K^{+}\right)+A\left(B^{+} \rightarrow \pi^{+} K^{0}\right) \\
& =-(\hat{T}+\hat{C}) e^{i \gamma}+\left(\hat{P}_{E W}+\hat{P}_{E W}^{C}\right)=-(\hat{T}+\hat{C})\left(e^{i \gamma}-q e^{i \phi} e^{i \omega}\right) .
\end{aligned}
$$

Here $A_{3 / 2} \equiv\left|A_{3 / 2}\right| e^{i \phi_{3 / 2}}$ is an isospin $I=3 / 2$ amplitude, where $\gamma=(70 \pm 7)^{\circ}$ is the corresponding UT angle, and $|\hat{T}+\hat{C}|$ can be determined from the $B \rightarrow \pi \pi$ system using the $S U$ (3) relation [9]:

$$
|\hat{T}+\hat{C}|=R_{T+C}\left|V_{u s} / V_{u d}\right| \sqrt{2}\left|A\left(B^{+} \rightarrow \pi^{+} \pi^{0}\right)\right| .
$$

The $S U$ (3)-breaking effects are given by $R_{T+C}=1.2 \pm 0.2$, where the central value is obtained in factorization and the uncertainty allows for non-factorizable corrections $[2,10]$.

The cleanest way to determine $\phi_{00}$ is from the amplitude triangles corresponding to the isospin relation for the neutral decays in Eq. (3.1). It requires only $S U(3)$ input from $R_{T+C}$ and $R_{q}$, and no topologies have to be neglected [2]. From Eq. (2.4), we can then determine $S_{\mathrm{CP}}^{\pi^{0} K_{\mathrm{S}}}$ as a function of $A_{\mathrm{CP}}^{\pi^{0} K_{\mathrm{S}}}$. There is a fourfold ambiguity in the determination of $\phi_{00}$, which can be resolved using the neutral $B \rightarrow \pi K$ data as discussed in Refs. [2,3]. Finally, we obtain the correlation shown in the left panel of Fig. 1, which is more constrained than in previous work [2] due to a better determination of $\gamma$. We observe a discrepancy between the data and the correlation at the $2.5 \sigma$ level.

In the right panel of Fig. 1, we show a new constraint, obtained from the angle $\phi_{ \pm} \equiv \arg \left(\overline{\mathrm{A}}_{ \pm} \mathrm{A}_{ \pm}^{*}\right)$ between $A_{ \pm} \equiv A\left(B_{d}^{0} \rightarrow \pi^{-} K^{+}\right)$and its CP-conjugate $\bar{A}_{ \pm}$. For $\phi=0^{\circ}$, which includes the SM, we 

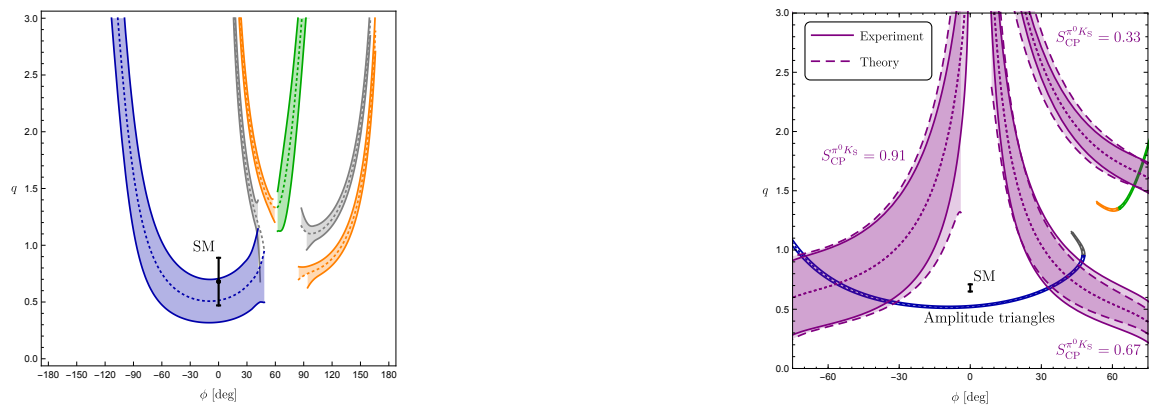

Figure 2: Contours in the $\phi-q$ plane for charged $B \rightarrow \pi K$ data following from the isospin relation in Eq. (3.1). The left panel shows current data, whereas the right one corresponds to future scenarios.

obtain $\left.\phi_{ \pm}\right|_{\phi=0}=2 r \cos \delta \sin \gamma+\mathscr{O}\left(r^{2}\right)=(8.7 \pm 3.5)^{\circ}$, where the numerical value follows from Eq. (2.2). We can also extract this angle from the amplitude triangles. The tension between these two constraints shows that also the correlation itself is not in agreement with the SM. We could obtain a consistent picture in Fig. 1 if the mixing-induced CP asymmetry of $B_{d}^{0} \rightarrow \pi^{0} K_{\mathrm{S}}$ moved up by $\sim 1 \sigma$ and $\mathscr{B} r\left(\pi^{0} K^{0}\right)$ went down by $\sim 2.5 \sigma$. On the other hand, Fig. 1 may be a hint of NP, where a modified EW penguin sector is a particularly interesting scenario.

\section{Determination of the Electroweak Penguin Parameters}

The isospin relation in Eq. (3.1) can also be used to obtain contours in the $\phi-q$ plane [3]. This analysis may be done for the neutral and charged decays separately. It requires us to fix the relative orientation of the triangles, which is done through $S_{\mathrm{CP}}^{\pi^{0} K_{\mathrm{S}}}$ in the case of the neutral decays, and with the angle between $A\left(B^{+} \rightarrow \pi^{+} K^{0}\right)$ and its $\mathrm{CP}$ conjugate, which is of $\mathscr{O}\left(1^{\circ}\right)$, for the charged decays. Since the current uncertainty of $S_{\mathrm{CP}}^{\pi^{0} K_{\mathrm{S}}}$ is still large [8], we perform the analysis for the charged decays, yielding the contours in the left panel of Fig. 2. This method requires $S U(3)$ input only to determine $|\hat{T}+\hat{C}|$ from Eq. (3.2), and no topologies have to be neglected.

In order to determine the values of $q$ and $\phi$ we need further information. This can be obtained by converting a measurement of $S_{\mathrm{CP}}^{\pi^{0} K_{\mathrm{S}}}$ into a value of $\phi_{00}$. Eq. (2.5) then yields a contour in the $\phi-q$ plane, using the hadronic parameters in Eq. (2.2). As the strong phases enter only as $\cos \delta_{(\mathrm{c})}$, this expression is very insensitive to variations of these parameters, thereby having a theoretically favourable structure. Furthermore, the small contributions from colour-suppressed EW penguins can be included through data [3].

In view of the large current uncertainty of $S_{\mathrm{CP}}^{\pi^{0} K_{\mathrm{S}}}$, we study three scenarios. In the right panel of Fig. 2, we give again the contours from the amplitudes triangles, now assuming perfect measurements and progress on the calculation of $R_{T+C}$ [2]. In addition, we show the contours from $S_{\mathrm{CP}}^{\pi^{0} K_{\mathrm{S}}}$, where we consider a precision of \pm 0.04 for the $\mathrm{CP}$ asymmetries of $B_{d}^{0} \rightarrow \pi^{0} K_{\mathrm{S}}$ at the end of Belle II [11], and include $20 \%$ non-factorizable $S U(3)$-breaking corrections for the hadronic parameters entering Eq. (2.5). We give separately the experimental and theoretical uncertainties, and observe that we can match the experimental precision with theory. Moreover, we see that $S_{\mathrm{CP}}^{\pi^{0} K_{\mathrm{S}}}$ provides complementary information on $q$ and $\phi$, allowing the determination of these parameters. 


\section{Conclusions}

We have performed a state-of-the-art $B \rightarrow \pi K$ analysis, finding a tension with the SM in the correlation of the $B_{d}^{0} \rightarrow \pi^{0} K_{\mathrm{S}} \mathrm{CP}$ asymmetries. In order to clarify this intriguing picture, either data have to move to confirm the SM, or we may have NP, where a modified EW penguin sector provides a particularly interesting scenario. We present a new strategy to determine the EW penguin parameters $q$ and $\phi$ with unprecedented precision at future $B$-physics experiments. It has the potential to resolve this puzzling situation and reveal new sources of $\mathrm{CP}$ violation.

\section{Acknowledgments}

I would like to thank R. Fleischer, E. Malami, and K. K. Vos for the enjoyable collaboration. This research has been supported by the Netherlands Organisation for Scientific Research (NWO).

\section{References}

[1] A. J. Buras, R. Fleischer, S. Recksiegel and F. Schwab, $B \rightarrow \pi \pi$, new physics in $B \rightarrow \pi K$ and implications for rare K and B decays, Phys. Rev. Lett. 92 (2004) 101804 [hep-ph/ 031225 9]; Anatomy of prominent $B$ and $K$ decays and signatures of $C P$ violating new physics in the electroweak penguin sector, Nucl. Phys. B 697 (2004) 133 [hep-ph/ 0402112$].$

[2] R. Fleischer, S. Jäger, D. Pirjol and J. Zupan, Benchmarks for the New-Physics Search through CP Violation in $B^{0} \rightarrow \pi^{0} K_{\mathrm{S}}$, Phys. Rev. D 78 (2008) 111501 [arXiv:0806.2900 [hep-ph] ].

[3] R. Fleischer, R. Jaarsma and K. K. Vos, Towards New Frontiers with $B \rightarrow \pi K$ Decays, arXiv:1712.02323 [hep-ph]; R. Fleischer, R. Jaarsma, E. Malami and K. K. Vos, Exploring $B \rightarrow \pi \pi, \pi K$ Decays at the High-Precision Frontier, arXiv:1806.08783 [hep-ph] .

[4] M. Neubert and J. L. Rosner, New bound on $\gamma$ from $B^{ \pm} \rightarrow \pi K$ decays, Phys. Lett. B 441 (1998) 403 [hep-ph/9808493]; Determination of the weak phase $\gamma$ from rate measurements in $B^{ \pm} \rightarrow \pi K, \pi \pi$ decays, Phys. Rev. Lett. 81 (1998) 5076 [hep-ph/9809311].

[5] M. Beneke, G. Buchalla, M. Neubert and C. T. Sachrajda, QCD factorization in $B \rightarrow \pi K, \pi \pi$ decays and extraction of Wolfenstein parameters, Nucl. Phys. B 606 (2001) 245 [hep-ph/ 0104110 ].

[6] R. Fleischer, R. Jaarsma and K. K. Vos, New strategy to explore CP violation with $B_{s}^{0} \rightarrow K^{-} K^{+}$, Phys. Rev. D 94 (2016) 113014 [arXiv: 1608.00901 [hep-ph] ]; Towards New Frontiers in the Exploration of Charmless Non-Leptonic B Decays, JHEP 1703 (2017) 055 [arXiv: 1612.07342 [hep-ph]].

[7] M. Gronau, A Precise sum rule among four $B \rightarrow K \pi$ CP asymmetries, Phys. Lett. B 627 (2005) 82 [hep-ph/ 0508047 ]; M. Gronau and J. L. Rosner, Rate and $C P$-asymmetry sum rules in $B \rightarrow K \pi$, Phys. Rev. D 74 (2006) 057503 [hep-ph/ 0608040 ].

[8] C. Patrignani et al. [PDG], Review of Particle Physics, Chin. Phys. C 40 (2016) 100001

[9] M. Gronau, J. L. Rosner and D. London, Weak coupling phase from decays of charged B mesons to $\pi K$ and $\pi \pi$, Phys. Rev. Lett. 73 (1994) 21 [hep-ph/9404282].

[10] A. Khodjamirian, T. Mannel and M. Melcher, Flavor SU(3) symmetry in charmless B decays, Phys. Rev. D 68 (2003) 114007 [hep-ph/ 0308297 ].

[11] T. Abe et al. [Belle-II Collaboration], Belle II Technical Design Report, arXiv: 1011.0352 [physics.ins-det]. 